Revista de Investigación Educativa 24

enero-junio, 2017 | ISSN 1870-5308 | Xalapa, Veracruz

Instituto de Investigaciones en Educación | Universidad Veracruzana

\title{
Estudio evaluativo de la escuela rural en la provincia de
} Cabinda, Angola

Evaluative Study of Rural School in the Province of Cabinda, Angola

\author{
Mtro. Fernando Bumba \\ Docente \\ Escola de Educação de Cabinda, Angola \\ felibumba@hotmail.com \\ Dra. María Pilar Cáceres Reche \\ Docente-Investigadora \\ Departamento de Didáctica y Organización Escolar \\ Universidad de Granada, España \\ caceres@ugr.es \\ Dra. Miriam Ágreda Montoro \\ Docente-Investigadora \\ Departamento de Pedagogía, Universidad de Jaen, España \\ magreda@ujaen.es
}




\section{Estudio evaluativo de la escuela rural en}

la provincia de Cabinda, Angola

Fernando Bumba, María Pilar Cáceres Reche, Miriam Ágreda Montoro

Este artículo presenta un estudio evaluativo de la escuela rural, específicamente de enseñanza primaria, en la provincia de Cabinda, Angola. El objetivo principal es conocer la situación actual del profesorado de las zonas rurales en educación primaria de Cabinda: el contexto, la infraestructura, los recursos y las condiciones en las que se desarrolla su labor docente. Para ello, se diseñó un estudio no experimental basado en la aplicación de un cuestionario, de escala tipo Likert, a los docentes de estas escuelas rurales. El análisis y la interpretación de los resultados obtenidos han permitido extraer inferencias, posibilitando la concreción de una serie de contribuciones relevantes y de importancia. Las escuelas rurales deben ser mejoradas tanto en infraestructura como en recursos y medios para facilitar un adecuado desempeño docente. Con ello, mediante una educación de calidad a toda la población escolar, se conseguirá la potenciación y el desarrollo de las zonas rurales.

Palabras clave: Educación primaria; demandas laborales; escuela rural; profesorado.

This article presents a study on the analysis of the working conditions of teachers in primary schools of Cabinda, Angola, located in rural areas of the province. The main objective is to know what are the resources provided to the schools for its development, e.g. canteens, put aid, teaching resources. To do so, was a non-experimental study based on a questionnaire of scale Likert-type teachers of these rural schools. The analysis and interpretation of the results obtained allowed to draw inferences, enabling the realization of a series of relevant contributions and importance. Rural schools should be improved both in infrastructure and in resources and means to facilitate an adequate teaching performance. Thus, by means of a quality education to the entire school population, the empowerment and development of rural areas will be achieved.

Keywords: Primary school; working needs; rural school; teachers. 


\title{
Estudio evaluativo de la escuela rural en la provincia de Cabinda, Angola
}

\author{
Evaluative Study of Rural School in the Province of Cabinda, Angola
}

\section{Introducción}

$\mathrm{D}$ esde la época de la colonización portuguesa, la figura del docente no ha tenido condiciones laborales adecuadas que faciliten su labor y mejoren la calidad educativa. Teniendo en cuenta esto, surge una serie de interrogantes como ¿cuáles son las condiciones laborales necesarias para el buen desempeño de la labor docente desde las exigencias impuestas por la globalización? y, concretamente, ¿qué demanda la escuela angolana atendiendo a su ubicación en el contexto rural de Cabinda?

Por ende, el origen de esta investigación nace a partir del objetivo primordial de conocer y analizar las condiciones laborales en las que se encuentran los maestros de las escuelas de enseñanza primaria de las zonas rurales de Cabinda, Angola.

Para Pedraza (2011), la escuela rural puede ser definida como una estructura singular, sobre todo debido a la situación geográfica en la que se encuentra. Caride (2014) afirma que la escuela es siempre escuela, ya sea grande o pequeña, agrupada o única, vieja o nueva. Sin embargo, contradiciendo la afirmación anterior y basándonos en el contexto de las escuelas rurales de Cabinda, debemos decir que las políticas socio-educativas portuguesas, la revitalización de las ideologías neoliberales y de la competitividad social han provocado y reforzado la consideración de la zona rural como un espacio residual, en contraposición con el mundo urbano en expansión, cada vez más enaltecido y globalizado (Caride, 2014).

Siendo así, dentro de nuestro contexto, sería inadecuado afirmar que todas las escuelas rurales son improvisadas y que no tienen recursos suficientes para el desempeño profesional de los docentes. Hoy en día sabemos que, en verdad, son escuelas que aúnan características típicas de contextos rurales impuestas por el dominio urbano.

En cuanto a las condiciones laborales del maestro rural de Cabinda, podemos decir que no son las adecuadas en su contexto genérico. Existen maestros que tienen 
que enfrentar diversas situaciones difíciles, como elevadas tasas de analfabetismo; la falta de alojamiento para docentes, que implica la necesidad de desplazarse todos los días desde su casa a la escuela; la antigüedad de las infraestructuras donde se sitúan las escuelas (algunas construidas por la propia población); la dificultad de acceso al centro escolar, etcétera. Además, no podemos obviar la falta de medios de transporte, que dificulta la movilidad y el desplazamiento en y entre las aldeas.

Actualmente, observamos la creciente importancia y relevancia de la integración de las redes sociales como base para la mejora del proceso de enseñanza-aprendizaje. Centrándonos en el contexto en el que se desarrolla nuestro estudio, nos damos cuenta de que el uso de las nuevas herramientas tecnológicas sería un punto clave para la modernización y promoción de un buen aprendizaje en las escuelas, así como la mejora y facilitación de la labor profesional del docente rural (Raso, Aznar \& Cáceres, 2014; Raso, Hinojo \& Sola, 2015).

Cabe destacar que el Gobierno de Angola ha tratado de mejorar la escuela rural en todas sus vertientes. No obstante, en algunas escuelas existen, todavía, edificios construidos y pertenecientes a la época anterior a la Independencia. Dichas infraestructuras se hallan en mal estado de conservación; incluso algunas fueron construidas con barro y otros materiales tradicionales de la época. No debemos obviar el hecho de que estas construcciones fueron fruto de la iniciativa y el esfuerzo del propio pueblo.

Pensamos que los centros escolares situados en las zonas rurales de Cabinda reúnen un conjunto de características específicas pertenecientes a la escuela rural. Tal y como recogen Libâneo, de Oliveira y Toschi (2005) y Delgado (2011), el gran desafío de la escuela es hacer del ambiente escolar un medio que favorezca al alumno en la adquisición de las mejores competencias y habilidades, dejando de ser meramente un lugar o punto de encuentro, para llegar a establecerse como un lugar de desarrollo constante que proporcione una construcción del aprendizaje de forma placentera y funcional. El gran problema que afecta al contexto educativo angolano se basa en la escasez de financiación y de recursos que son destinados a educación y, en menor medida, al contexto de las escuelas de nivel primaria de las zonas rurales de Cabinda. 


\section{La educación primaria en el contexto angoleño}

Situándonos en años anteriores al proceso de Independencia, nos encontramos un sistema educativo angoleño de escasa funcionalidad. En los años setenta se vislumbraba una tasa de escolarización inferior al $35 \%$ y la tasa de alfabetización no superaba el 10\%. Unido a esto la cualificación del profesorado dedicado a la educación primaria era escasa o nula; además la mayoría de las escuelas se encontraba situada en zonas urbanas (Angulo, 2004; Cardoso, 2015).

También era significativa la separación existente entre las escuelas normalizadas; se da una dicotomía entre aquellos centros en los que sólo se permitía la entrada a alumnado mestizo y blanco, y aquellos donde se admitía al alumnado negro, pues en este último caso se trataba de escuelas con una calidad cuestionable. La apertura de las escuelas públicas propició el aumento de la tasa de escolarización, sobre todo en las zonas urbanas. Las escuelas rurales de educación primaria tuvieron la necesidad de unirse a los centros escolares de misioneros; aun así, la calidad educativa era mucho menor (Cardoso, 2016).

Según un informe de la Organización Internacional del Trabajo en 2010, de los diez millones y medio de niños y niñas que se encontraban en una situación de trabajo forzoso, 17\% pertenecía al continente africano. Específicamente en Angola, según un estudio de Fondo de las Naciones Unidas para la Infancia (UNICEF, 2013), uno de cada cuatro niños debe trabajar para cubrir las necesidades familiares; esta realidad va en contra de la legislación del país, que prohíbe el trabajo en menores de 14 años.

En los últimos datos arrojados por los informes de la Organización de las Naciones Unidas para la Educación, la Ciencia y la Cultura (UNeSCO, 2013) y UNICEF (2013) $40 \%$ de la población angolana se encuentra no escolarizado. Una de las causas precisamente es la escasez de escuelas en las zonas remotas del territorio, sumando la escasez de transporte, falta de instalaciones sanitarias, aulas deplorables, infraestructuras en semi-ruina o arruinadas completamente y la baja capacitación docente.

En el contexto angolano y con la reforma del sistema educativo, la educación primaria se entiende como el primer nivel de enseñanza básica formal y se inicia en $4^{\circ}$ grado (antes de la reforma de 2001). Son admitidos en este nivel los niños con edades comprendidas entre los 6 y 14 años, es gratuito y obligatorio (Zassala, 2012). Ya en la época de la reforma educativa, la educación primaria se convirtió en el punto de partida para el avance por los demás niveles educativos. Consta de 6 años unificados y tiene como objetivo asegurar la preparación del alumnado para la continuación hacia y por la en- 
señanza secundaria. Todo lo mencionado se refleja en los artículos 8 y 17 de la Ley ${ }^{\circ}$ 13/2001 (Comisaão de Acompanhamento das Acçõoes da Reforma Educativa, 2009).

La escuela ofrece una enseñanza general que comprende desde la educación formal hasta la educación de adultos, según el art. 17 de la Ley n ${ }^{\circ}$ 13/2001, y abarca desde $1^{\circ}$ grado hasta $6^{\circ}$. Esta normativa se hace extensible a las escuelas que se encuentran dentro de las comunidades rurales. Este hecho se convierte en nuestra mayor preocupación, ya que las zonas rurales se sitúan lejos de las urbanas donde las leyes son promulgadas.

\section{La realidad de la Educación Primaria en el contexto de otros países de África}

Generalmente, el tema de la educación ha provocado una serie de situaciones problemáticas en cualquier territorio africano. Haciendo un recorrido por la historia y vida africana, destacamos el seminario llevado a cabo en Níger en el año 1989. Fue patrocinado por el Instituto Internacional de Planificación Educativa y arrojó resultados significativos a partir de los diagnósticos presentados por los participantes, todo ellos con el mismo punto de partida: la base del sistema educativo, la escasez de formación del profesorado y la deficiencia en las partidas económicas destinadas a las escuelas y la educación en general (Zassala, 2003).

La Educación Primaria logró una notable expansión durante los años 60 y 70, pero es a partir de los años 80 cuando se comienza a observar un declive generalizado de la eficacia educativa en la mayoría de los países representados. Este fenómeno estaba íntimamente relacionado con una serie de factores, sobresaliendo el bajo rendimiento económico, las guerras y otros subyacentes.

En algunos países de África, la enseñanza primaria sigue rigiéndose por normas y reglas que se instauraron y pertenecen a la época colonial, tiempo en el que estos territorios no eran independientes. Este hecho puede ejemplificarse con la República Popular y la República Democrática del Congo, donde más allá del examen realizado por la UNESCO que les da credibilidad como Estado, nos encontramos con una enseñanza no gratuita. Desde hace mucho, las lenguas nacionales en las escuelas oficiales se convirtieron en un gran vehículo para el aprendizaje de la lengua oficial extranjera; esta es una de las características que marcan diferencias entre la enseñanza primaria de Angola respecto a otros países vecinos. 
Otra ejemplificación que nos ayuda a clarificar nuestra reflexión es la de la reforma llevada a cabo en Mali. Poco después del logro de la independencia en 1960, la República de Mali priorizó de manera absoluta la necesidad de una profunda reforma del sistema educativo, ya que había sido heredado del régimen colonial. Para ello se hizo un llamamiento generalizado, no sólo al personal educativo, sino a todos los poderes nacionales (Zassala, 2003). De forma semejante, Angola eliminó todos los puntos en común con la antigua educación colonial y se trasladó a otras realidades; por consiguiente, se ha generado una fuente de desequilibrio en la enseñanza básica angoleña en su reafirmación como territorio independiente. Volviendo a las características de la escuela en Mali, los centros de enseñanza primaria o básica fueron sustituidos por la nueva "escuela fundamental", que comprende un primer ciclo de seis años y un segundo de tres años. El alumnado no sería alejado de su familia antes de los 15 o 16 años, premisa subyacente en todas las comunidades de la República.

En Ruanda, la enseñanza primaria no solamente sirve para conducir al alumnado a la etapa de la educación secundaria, sino que también pretende dotar a la nación de unos ciudadanos responsables y capaces de participar en el esfuerzo general de la sociedad y para su desarrollo (Haguma \& Murenkzi, citado en Zassala, 2003). Concretamente, en el sistema actual, las enseñanzas comienzan en la escuela básica a los 7 años. Durante un total de seis años, es decir, dos ciclos de tres (tipo $3+3$ ), el alumnado se prepara para su futuro ingreso en la enseñanza secundaria, poniendo el acento en la formación general. Es importante destacar que el acceso a la enseñanza secundaria se realiza a través de un examen de selección en el sexto año de la etapa. A este respecto, el porcentaje máximo de estudiantes que son admitidos en la Educación Secundaria, una vez realizado el examen, es de $8 \%$. Estas estadísticas reflejan cierta mejoría dentro del sistema educativo. Como suele decirse, los mejores métodos nacen de unas buenas prácticas educativas. Luego, si a la escuela angoleña la aqueja la falta de estos elementos, la enseñanza se torna inconsistente. La situación ideal es ser partícipes de y en las buenas prácticas y experiencias educativas, sobre todo porque la enseñanza primaria es la base sustentadora de todas las demás etapas educativas.

A lo largo de este desarrollo se ha ido confirmando que la realidad educativa africana no tiene muchos puntos y características divergentes entre los diferentes territorios, sobre todo si hablamos y nos centramos en el ámbito educativo. En la mayoría de los países se comprueba que las condiciones laborales no son del todo adecuadas ni buenas, principalmente en las zonas o comunidades rurales. Por tanto, este es nuestro foco y objetivo de estudio. 


\section{La escuela rural en Cabinda. Una aproximación al objeto de estudio}

\subsection{Conceptualización}

Desde la literatura científica es difícil y compleja la tarea de acotar o definir el concepto de escuela rural (Raso, 2012). No sólo porque el término rural carga en sí una visión que engloba aspectos eclécticos, multidimensionales y consideraciones interminables, sino que también, siguiendo a Berlanga (2003, citado en Raso, 2012) "no es posible dar una definición universal y permanentemente válida de rural o urbano, sino tener [sic] una visión de conjunto con el fin de no caer en una dicotomía simplista porque ambos están en constante cambio" (p. 66). Los cambios que refieren a estos modelos pueden darse tanto en términos cuantitativos como cualitativos. Se entiende que los centros escolares reúnen un conjunto de formas de enseñanza específicas de estas zonas. A continuación se mencionan algunas de ellas (Cantón, 2004, p. 106):

- Baja relación alumno-maestro.

- La forma de agrupación no es por grado.

- En última instancia, son los únicos servicios públicos en la localidad.

- Tienen ciertas dificultades para acceder a la propiedad cultural causando algún aislamiento en sus alumnos.

Para el caso que nos ocupa, la escuela rural en Cabinda, destacamos que presenta diferencias respecto a la urbana. Estas diferencias son más acusadas respecto a otros países. La zona rural es un espacio que se encuentra situado en las zonas más distantes de las ciudades, es decir, se establecen como municipios no clasificados. Pueden ser espacios no urbanizados cuyas principales actividades son la agricultura, la avicultura, la pesca y la caza. Desde luego, cuando se discuten propuestas educacionales acordes a los tiempos de regeneración en los que vivimos, frecuentemente se pone en duda cuál sería el currículo que se adecua a las escuelas rurales, sobre todo por la diversidad existente entre ambas (Trevizan, 2003). La supremacía del medio urbano sobre el rural es un problema que viene dado históricamente.

En la actualidad se hace necesario promover un "desarrollo sostenible", tal y como se apunta desde el Informe Brundtland de la ONU (1987). Un desarrollo capaz de cubrir las necesidades del presente sin comprometer la capacidad de las generaciones futuras, apoyándose en tres pilares básicos: económico, social y financiero (Callado, Molina, Pérez \& Rodríguez, 2015; Morales, 2014). 
Por otra parte, es necesario considerar que la escuela rural de Cabinda necesita tanto de las condiciones básicas en el contexto laboral (recursos, transportes, infraestructuras) como, y no menos importante, de recursos tecnológico, ya que ayudarían a promover acciones y desmitificar la imagen confusa de la escuela como centro única y exclusivamente de alfabetización. Así pues, las Tecnologías de la Información y la Comunicación (TIC) como recurso didáctico y organizativo pueden, con la debida integración curricular, ofrecer un conjunto de posibilidades a una nueva escuela rural (Llorente, Barroso \& Cabero, 2015; Raso, Hinojo \& Sola, 2015; Sánchez \& Navarro, 2016).

Centrándonos en la diferencia entre la escuela rural y la urbana, puede ser entendida cuando lleguemos a definir las dos comunidades educativas. Se sintetizará qué tipo de enseñanza y/o escuela existe en cada una de las ubicaciones. Es importante apuntar que la escuela, independientemente de la zona en la que se encuentre, nunca ha tenido la fuerza, coraje y posibilidad de manifestar a la administración pública esa gestión precaria. No es difícil comprender que en las zonas rurales se dé la existencia de una elevada tasa de abandono escolar y los edificios sean mucho peores que los urbanos. Además, la familia tiene que hacer frente a las reparaciones de esas construcciones que actúan como escuelas, a pesar de las dificultades económicas en las que pueden llegar a encontrarse. Existe una muestra patente de la falta de solidaridad de la administración respecto a las idiosincrasias de las zonas rurales.

Creemos que una escuela rural debería configurarse como el centro formativo de una comunidad y no marcar diferencias según la zona geográfica en la que se sitúa. Debe suprimirse esa relación dependiente que existe sobre que el entorno urbano presenta una mayor calidad educativa. Siguiendo a Raso, Aznar y Cáceres (2014) hemos constatado que el medio rural ha sido un reducto subdesarrollado que se percibe como un paraíso de tranquilidad, destinado al turismo rentable cuando se quiere huir de la agitación de la ciudad. Para los autores, el hombre de campo es visto como un ser alternativo.

En términos generales, la República de Angola manifiesta tener un gran desafío para modernizar y cambiar el medio rural a través de proyectos educativos. Sin embargo, a pesar de todo el esfuerzo realizado, aún encontramos un nivel de calidad deficiente en las escuelas rurales; sobre todo, desde el punto de vista de la creación de las condiciones necesarias y adecuadas, atendiendo a la funcionalidad y provisión de recursos fundamentales para la mejora de la calidad del proceso de enseñanzaaprendizaje. 
Las limitaciones más acusadas que se presentan en el día a día en estas escuelas de la región son la falta de iluminación eléctrica, agua. Más grave es que se "castiga" al profesorado de centros urbanos que cometen "actos de indisciplina" cuando se les destina a la escuela rural. En este sentido, es claramente observable la imagen tan negativa que suele tener el contexto no urbano, al cual nadie quiere ser trasladado y en el que se dificulta el cambio y mejora continuamente (Bustos, 2011; Callado et al., 2015; Vázquez, 2016).

\subsection{Contextualización}

Para hablar de las escuelas rurales de Cabinda es necesario situarlas en el contexto angoleño. Angola es un país con capital en Luanda, con una superficie de $1,246,700 \mathrm{~km}^{2}$ y una población estimada de 24,383,301 habitantes. Se extiende a lo largo de 18 provincias (Ceita, 2014) y tiene el portugués como idioma oficial. Existe una moneda única llamada kwanza. Geográficamente, Angola está situada en la costa Atlántica Sur de África Occidental, entre Namibia y el Congo. También hace frontera con la República Democrática del Congo y Zambia, al oriente. El país está dividido entre una franja costera árida y una sabana seca en el interior sur y sudeste; la floresta tropical se encuentra en el norte de Cabinda.

En el contexto económico, la guerra civil surgida en el país afectó gravemente a la economía, lo que provocó un declive socio-económico, relegándola al nivel más alto de pobreza respecto a otros territorios. En este momento, Angola presenta buenos índices de recuperación económica apoyada por sus exportaciones de petróleo y otros recursos minerales.

El foco de nuestro estudio, la provincia de Cabinda, pertenece a las 18 provincias de Angola. Es un enclave limitado al norte con la República del Congo (Brazavile), al este y al sur con la República Democrática del Congo y, finalmente, con el océano Atlántico al oeste. Su capital es Cabinda, conocida como Tchiowa, y posee una superficie de $7,283 \mathrm{~km}^{2}$ y con una población cercana a los setecientos mil habitantes (Ceita, 2014). La población pertenece, casi en su totalidad, a los pueblos de Bantu, concretamente al grupo fiote, conjunto específico etnolingüístico compartido por la población.

Cabinda sirve de capital administrativa de la provincia y fue considerada como ciudad a partir del 28 de mayo de 1956. La provincia posee cuatro municipios llamados Cabinda, Cacongo, Buco-Zau y Belize. Tiene ocho comunas, con un clima tropi- 
cal húmedo, precipitaciones anuales en torno a los $800 \mathrm{~mm}$, y su temperatura media anual varia entre los 25 y los $30^{\circ}$ grados.

\subsection{Situación socio-económica y cultural}

La situación social de la población de Cabinda, desde nuestra perspectiva, ha sido siempre difícil de caracterizar. Después de un largo conflicto armado, en el que se encontró inmersa durante más de tres décadas, el gobierno ha intentado buscar soluciones para mejorar las condiciones de vida de la población. Fijándonos en el contexto social y la condición de la población, se destaca que el desarrollo aún no ha sido alcanzado según previsiones del programa de gobernación angolana.

En Resenha social (2013) se afirma que la población de Cabinda presenta un alto riesgo social porque alumnado y docentes aún trabajan en condiciones extremadamente precarias. Para confirmar esta visión, en 2015 acudimos a varias escuelas rurales de la provincia y constatamos las dificultades en las que se encuentran inmersos. Se visitaron escuelas situadas en el Alto Maiombe (Municipio de Buco-Zau, Micuma I); municipio de Belize, comuna del Miconge que dista $280 \mathrm{~km}$ al norte de Cabinda; se visitaron también las comunas de Necuto y Dingue (aldea de Beira Nova) y el municipio sede de la provincia (aldea de Chiweca). Estos son sólo algunos lugares en los que se ha podido constatar las problemáticas sociales y educativas de esta población.

El Jornal de Angola ${ }^{1}$ afirma, además, que algunas personas consideran que la enseñanza practicada en las aldeas y comunas es anti-pedagógica porque la manera en la que se desarrolla recuerda a una comunidad primitiva.

Por otro lado, la agricultura, la pesca y la caza volvieron a ser de subsistencia, sobre todo cuando se reanudó su práctica en 2011. A esto unimos el crecimiento de la industria petrolífera, que representó el 70\% del total de ingresos en el año 2010. La industria de la extracción de diamantes también es un factor económico importante a tener en cuenta.

En lo que se refiere a la cultura, Cabinda posee determinadas peculiaridades: formas de vestir, comer, formas de andar y hablar, rituales culturales. El Museo de la provincia es uno de los mayores centros de investigación y colección de la tradición del pueblo. El centro cultural Chiluango es la institución que cuida y desarrolla diversas actividades en las áreas de música, danza y artes escénicas.

1. Jornal de Angola (http://jornaldeangola.sapo.ao) 


\subsection{Condiciones materiales e infraestructura}

Desde 2008 hasta 2015 el número de escuelas de calidad aceptable no se corresponde con las expectativas de la población, tampoco refleja el esfuerzo del gobierno por ampliar la red escolar en zonas rurales. Esto es consecuencia del conflicto armado que provocó la huida de los habitantes y que ahora se están reintegrando de nuevo. Nos centramos en aquellas escuelas rurales que se encuentran fuera de las villas y que no se benefician de ningún servicio social y viven sin luz ni agua.

Si hablamos de escuela rural es imposible pensar en centros que tengan recursos e infraestructuras adecuadas situados fuera de las villas. En lo que respecta a las condiciones laborales de los maestros en este ámbito, adquieren relevancia el salario, los espacios físicos y la infraestructura, los recursos tanto materiales como tecnológicos, el estado anímico de los docentes, etcétera, pues aportan posibilidades al correcto desempeño profesional y garantizan un clima efectivo en la escuela (Granados, Antolínez \& Rivera, 2014).

La planificación arquitectónica de los centros rurales de Cabinda no tiene más de tres o cuatro aulas y ni siquiera posee en total cinco alumnos por clase. El material didáctico utilizado no es el ideal para buenas prácticas y la labor educativa. Lógicamente hay una escasez de libros, cuadros, mapas.

\section{Diseño de la investigación}

\subsection{Problema y objetivos}

Tal y como se recoge en la primera parte de este artículo, el problema de investigación queda determinado como el estudio empírico de la situación actual del profesorado en las escuelas rurales de Cabinda. Para ello, el estudio queda articulado según los siguientes objetivos específicos:

a. Analizar las condiciones laborales de los maestros en las escuelas rurales de Cabinda, Angola.

b. Caracterizar las condiciones laborales, desde del punto de vista de infraestructura, ambiental, económica y funcional de los maestros de estas escuelas.

c. Proponer algunas líneas de actuación que puedan ayudar a mejorar el trabajo del maestro en el medio rural angolano. 


\subsection{Metodología e instrumento}

Cada proceso de investigación puede caracterizarse como un proceso de espiral donde cualquier resultado ofrece nuevas perspectivas y promueve nuevos estudios y nuevas investigaciones (Buendía \& Colás, 1998; Latorre, Del Rincón \& Arnal, 1996).

Así, para abordar la situación laboral de la figura del maestro en el contexto de las escuelas rurales de Cabinda, se utilizó una metodología descriptiva y con un diseño no experimental por método de encuesta, a través de la aplicación de un cuestionario. Esto nos permitió el conocimiento de la realidad de un modo más próximo y comprensivo a tenor del propio contexto.

Para la ejecución de este estudio, como se comentaba anteriormente, se hizo uso de un cuestionario construido y diseñado ad hoc, con 62 ítems y escala tipo Likert de cuatro opciones, que fueron divididos en dos grandes bloques:

a. Estructura abiótica: configuración arquitectónica de la escuela (24 ítems).

b. Contexto situacional: dividido en condiciones materiales ( 16 ítems), condiciones funcionales (ocho ítems), relaciones interpersonales (ocho ítems) y contribución a la comunidad (siete ítems).

Además se añade una dimensión de datos personales y profesionales del profesorado (edad, categoría profesional, años de experiencia, género). Para la validación a nivel estructural del instrumento, el contenido fue formalmente sometido a un juicio por criterio de expertos, un total de seis profesores titulares pertenecientes a los departamentos de Métodos de Investigación y Diagnóstico en Educación, Didáctica y Organización Escolar y Psicología. Para el cálculo del grado de consistencia interna y fiabilidad fue utilizado el coeficiente de Cronbach, cuyo valor ha sido alto (o.988).

\subsection{Población y muestra}

La población objeto de esta investigación está constituida por 374 maestros de las escuelas públicas de enseñanza primaria de las zonas rurales, de la cual se extrajo una muestra de 151 usando la estratificación aleatoria simple, cuya condición indispensable fue que todos ellos tuvieran la misma posibilidad de formar parte de la investigación por poseer características similares, conforme a la Tabla 1. 
Tabla 1. Población y muestra

\begin{tabular}{lccc}
\hline \multicolumn{3}{c}{ Municipios } & \multicolumn{3}{c}{ Población } \\
\hline & M & F & MF \\
\hline Belize & 25 & 05 & 30 \\
\hline Buco-Zau & 93 & 13 & 106 \\
\hline Cacongo & 46 & 10 & 56 \\
\hline Cabinda & 180 & 02 & 182 \\
\hline TOTAL & 344 & 30 & 374 \\
\hline
\end{tabular}

\begin{tabular}{ccc}
\hline \multicolumn{3}{c}{ Muestra } \\
\hline $\mathbf{M}$ & F & FM \\
\hline 25 & 05 & 30 \\
\hline 49 & 13 & 62 \\
\hline 31 & 10 & 41 \\
\hline 13 & 05 & 18 \\
\hline 118 & 33 & 151 \\
\hline
\end{tabular}

Fuente: Governo Provincial de Cabinda (2014).

\section{Discusión}

Teniendo en cuenta el primer objetivo, relacionado con el análisis centrado en la situación actual de los maestros rurales de Cabinda, queda patente que la configuración arquitectónica, las condiciones funcionales, interpersonales y materiales no benefician ni permiten la mejora de la labor del profesorado, requiriendo mejorarlas en su contexto genérico, ya que todos los maestros coincidían en su nivel de respuesta al respecto.

El segundo objetivo de nuestro trabajo relaciona la caracterización de esas condiciones, por lo que es posible distinguir los siguientes aspectos:

a. Las condiciones de las aulas necesitan ser mejoradas. Más de la mitad de docentes $(63.6 \%)$ considera que es fundamental este aspecto para atraer al maestro, posibilitar un buen trabajo y que los alumnos puedan desarrollar un aprendizaje de calidad (Tabla 2).

b. En cuanto a la existencia de los medios de transporte para los docentes en las escuelas rurales, los maestros muestran una gran insatisfacción por la falta de estos para llegar al centro escolar, destacando con un porcentaje elevadísimo del 85.6\% (Figura 1). 
Fernando Bumba, María Pilar Cáceres Reche, Miriam Ágreda Montoro

Tabla 2. La escuela posee aulas en perfectas condiciones

\begin{tabular}{llcccc}
\hline \multirow{2}{*}{ Respuestas } & Frecuencia & $\begin{array}{c}\text { Porcentaje } \\
\%\end{array}$ & $\begin{array}{c}\text { Porcentaje } \\
\text { válido }\end{array}$ & $\begin{array}{c}\text { Porcentaje } \\
\text { acumulado }\end{array}$ \\
\hline & $\begin{array}{l}\text { Totalmente en } \\
\text { desacuerdo }\end{array}$ & 69 & 45.7 & 45.7 & 45.7 \\
\cline { 2 - 6 } & En desacuerdo & 27 & 17.9 & 17.9 & 63.6 \\
\cline { 2 - 6 } & \begin{tabular}{l} 
De acuerdo \\
\cline { 2 - 6 }
\end{tabular} & 20 & 13.2 & 13.2 & 76.8 \\
\hline & $\begin{array}{l}\text { Totalmente de } \\
\text { acuerdo }\end{array}$ & 35 & 23.2 & 23.2 & 100.0 \\
\cline { 2 - 6 } & Total & 151 & 100.0 & 100.0 & \\
\hline
\end{tabular}

Fuente: Elaboración propia.

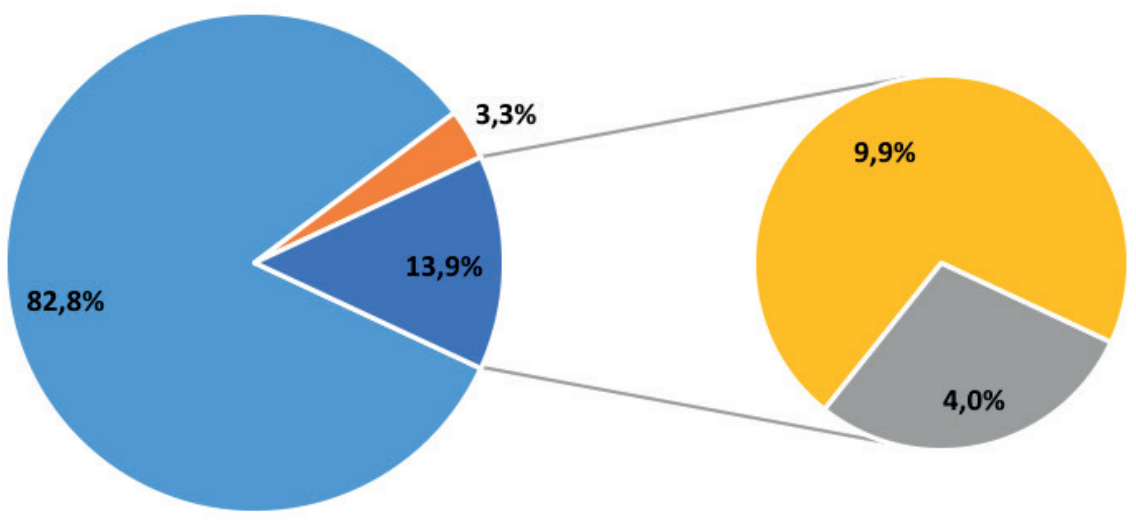

- Totalmente en desacuerdo $\square$ En desacuerdo $\square$ De acuerdo $\quad$ Totalmente de acuerdo

Figura 1. Existen medios de transporte para los empleados de la escuela

Fuente: Elaboración propia.

c. En la Figura 2 tenemos los resultados recogidos sobre las condiciones de trabajo para los maestros en las escuelas primarias rurales. Casi $80 \%$ de los docentes considera que los centros escolares donde trabajan no presentan buenas condiciones laborales. Todo indica que las condiciones existentes no son tan adecuadas como se desea para una buena práctica del trabajo docente. 


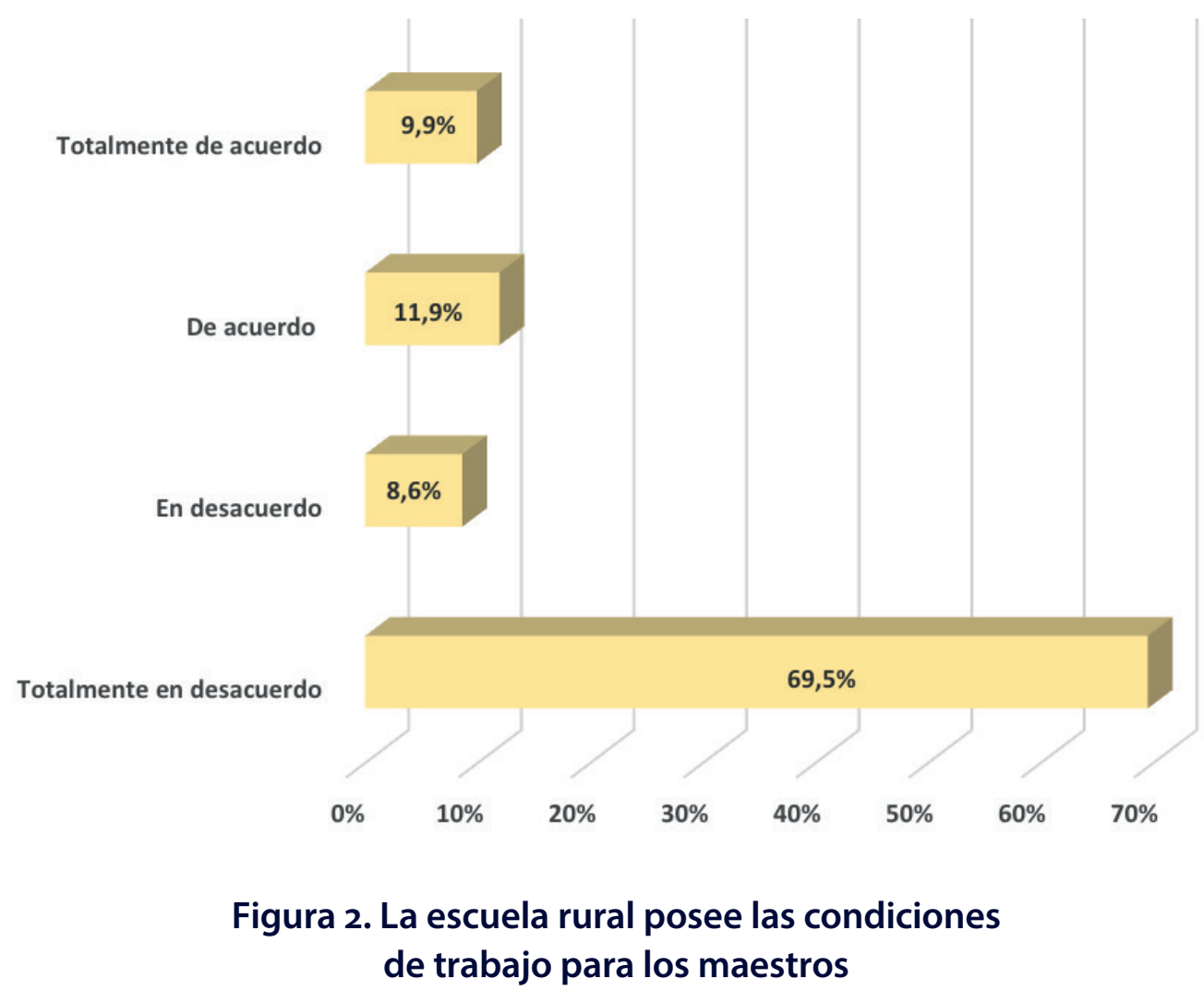

Fuente: Elaboración propia.

d. En relación al comedor escolar para la merienda de maestros y alumnos, fundamental para el desarrollo laboral, al tener que desplazarse largas distancias desde el domicilio hasta el centro, prácticamente la mayoría coinciden en que no poseen este tipo de instalaciones (93.4\%) (Figura 3). En lo que se refiere al puesto de socorro próximo a la escuela, también de gran importancia ante cualquier incidente que requiera una intervención desde la acción sanitaria, se añaden más dificultades, ya que sólo $15 \%$ afirma tener un puesto de socorro relativamente cerca al centro. Sin embargo, la mayoría de docentes coincide en apuntar su inexistencia (84.5\%). En cualquier caso, aunque no es competencia del Ministerio de Educación proporcionar tales condiciones sanitarias a la población, sí sería necesario que existiera una colaboración entre el Ministerio de Salud y el de Educación, en función de la situación subyacente (Figura 3). 
Fernando Bumba, María Pilar Cáceres Reche, Miriam Ágreda Montoro

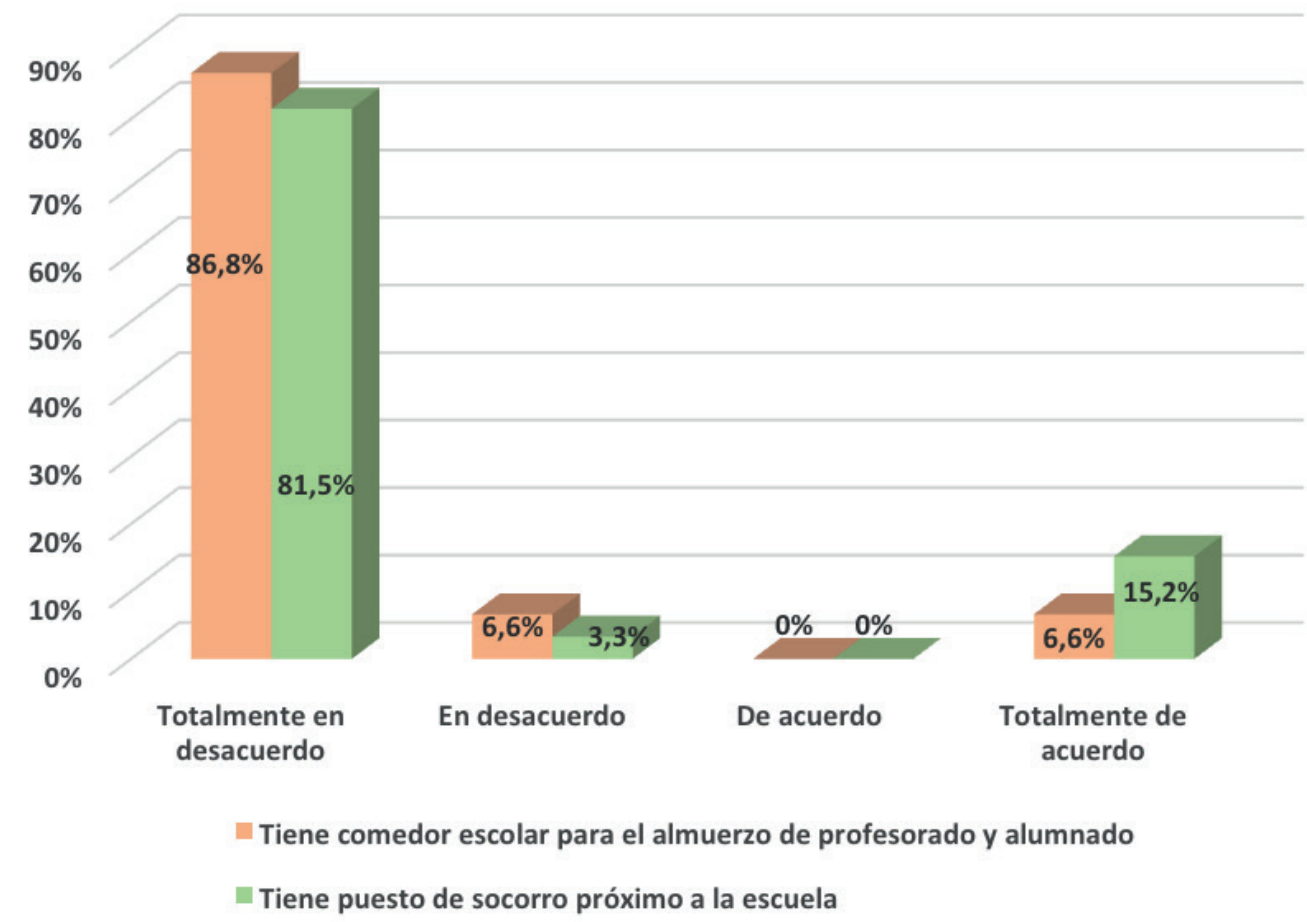

\section{Figura 3. Comparación sobre la existencia de comedor escolar y puesto de socorro escuelas rurales}

Fuente: Elaboración propia.

e. A pesar de la prácticamente inexistente presencia de comedor escolar, puesto de socorro, etcétera, en lo relacionado con el reparto y la distribución de manuales (libros) para el alumnado, más de la mitad de los docentes (63.6\%) afirma que recibe este material didáctico en el centro como medio de apoyo escolar para el alumnado sin recursos en estas zonas tan desfavorecidas. No obstante, aún existe $36.5 \%$ al que no se le facilita este tipo de materiales didácticos, por lo que es necesario aplicar medidas igualitarias para toda la población y los diferentes centros educativos de esta región angolana (Figura 4). 


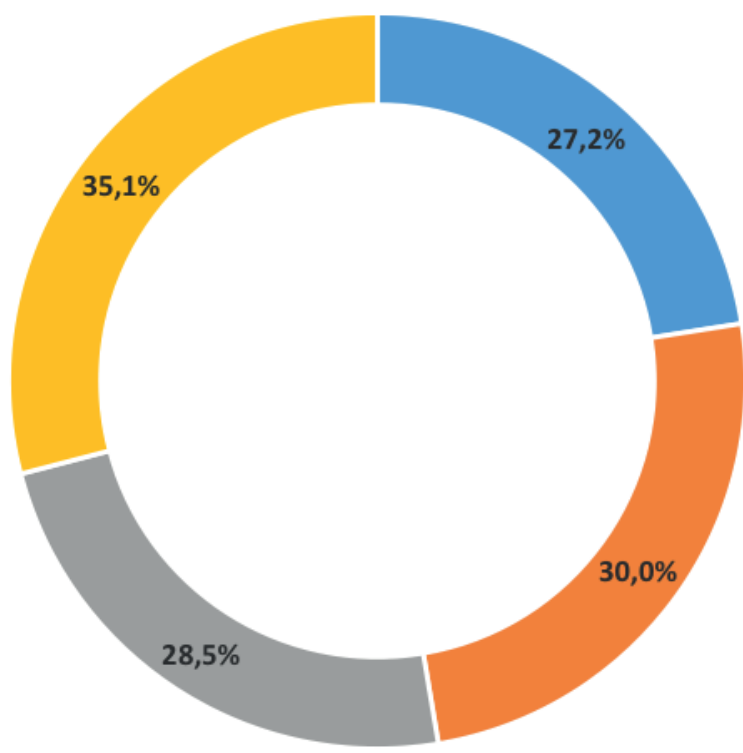

- Totalmente en desacuerdo $\quad$ En desacuerdo $\|$ De acuerdo $\quad$ Totalmente de acuerdo

Figura 4. La escuela proporciona los manuales (libros) para el alumnado

Fuente: Elaboración Propia.

\section{Conclusiones}

Teniendo en cuenta los objetivos iniciales del trabajo, así como en base a los resultados analizados, podemos destacar como conclusiones más significativas las siguientes:

- Las aulas de las escuelas rurales de Cabinda no reúnen las condiciones laborales necesarias para desarrollar una adecuada acción educativa, de acuerdo con las condiciones materiales, recursos sanitarios, didácticos, señalados por los propios docentes. Así pues, se observa la coincidencia, tal y como ocurre en muchos otros países y centros, con la "ignorancia" hacia "lo rural" y la necesidad de incorporar recursos y apoyos para su desarrollo y potenciamiento.

- En cuanto a la existencia de medios de transporte para apoyar a los maestros y otros empleados, afirman que no existen, por lo que todos aquellos maes- 
tros que no disponen de alojamiento en estas zonas, son forzados a desplazarse diariamente durante muchas horas hacia el lugar de trabajo, lo que implica un desgaste de tiempo, esfuerzo y dinero que perjudica de la estabilidad laboral del docente y su desempeño profesional. Para evitar esta situación, se podrían plantear medidas como la creación de un "bono" escolar para que el maestro y el alumno puedan tener derecho a viajar, acordando anualmente un valor simbólico con la empresa de transportes.

- Las instalaciones de las escuelas rurales no se adaptan tampoco a las necesidades biológicas de su población escolar, carecen de servicios básicos como el comedor escolar. También hay ausencia de un puesto de socorro cercano al centro, al menos para aplicar unos primeros auxilios que en ocasiones pueden llegar a salvar muchas vidas, sobre todo en los primeros minutos decisivos hasta que la persona es atendida por un médico. En este sentido, es fundamental por parte de las autoridades que se planteen impulsar el medio rural y, por tanto, la educación que se imparte, a través de una mayor financiación y dotación de recursos (comedor escolar, puesto de socorro, residencia escolar, biblioteca escolar y otros materiales didácticos) para garantizar unas condiciones laborales dignas para el desarrollo de un buen aprendizaje por parte de los alumnos y crear un clima motivador y de estabilidad laboral, que repercutirá en un mejor desempeño profesional por parte del docente.

\section{Lista de referencias}

Angulo, G. (2004). La Educación en Angola: Escenario y perspectiva para la colaboración española. Manuscrito inédito, Oficina Técnica de Cooperación, Angola.

Buendía, L., \& Colás, P. (1998). Métodos de investigación en Psicopedagogía. Madrid: Mc Graw-Gill Interamericana de España.

Bustos, A. (2011). Enseñar en la escuela rural aprendiendo a hacerlo. Evolución de la identidad profesional en las aulas multigrado. Revista de currículum y formación del profesorado, 11(3), 1-26.

Callado, J. A., Molina, M. D., Pérez, E., \& Rodríguez, J. (2015). La Educación Inclusiva en los colegios de zonas rurales. New Approaches in Educational Research, 4(2), 115-123. Doi: 10.7821/naer.2015.4.120. Recuperado de http://naerjournal.ua.es/ article/view/v4n2-6 
Cantón, I. (2004). Intervención organizativa en la Sociedad del Conocimiento. Granada: Grupo Editorial Universitario.

Cardoso, J. (2016). Realidades de la educación rural en Angola. Un estudio de caso. Revista Santiago, 140, 430-441.

Caride, J. A. (2014). A escola (rural) como pasado e futuro. Innovación Educativa, 24, 155-172.

Ceita, C. (2014). Resultados preliminares do recenseamento geral da população e da habitação de Angola 2014. Luanda: Instituto Nacional de Estatística. Recuperado de http://aiangola.com/wp-content/uploads/2016/o3/Publicação-Resultados-Definitivos-Censo-Geral-2014 Versão-22032016_DEFINITIVA-18H17.pdf

Comisaão de Acompanhamento das Acçõoes da Reforma Educativa. (2009). Informação sobre a Implementação do Novo Sistema de Educação-Reforma Educativa do Ensino Primário e Secundário. Luanda: Instituto Nacional de Bolsas de Estudo.

Delgado, M. (2011). Organización y gestión de centros y contextos educativos. Madrid: Universitas.

Fondo de las Naciones Unidas para la Infancia. (2013). Situação mundial da infância 2013: crianças com deficiência. Recuperado de http://www.unicef.org/brazil/ pt/resources 25542.htm

Governo Provincial de Cabinda (2014). A Província por Dentro. Recuperado de http://www.cabinda.gov.ao/Institucionais/QuemEQuem.aspx

Granados, N. P., Antolínez, N. M., \& Rivera, J. L. (2014). Incidencia de las condiciones laborales rurales en el clima de aula. Quaestiones Disputatae: temas en debate, 7(15). Recuperado de: http://revistas.ustatunja.edu.co/index.php/ qdisputatae/article/view/838

Latorre, A., Del Rincón, D., \& Arnal, J. (1996). Bases metodológicas de la investigación educativa. Barcelona: Hurtado.

Libâneo, J. C., de Oliveira, J. F., \& Toschi, M. S. (2003). Educação escolar: políticas, estrutura e organização. Sao Paulo: Cortez.

Llorente, M. C., Barroso, J., \& Cabero, J. (2015). Las tecnologías de la información y la comunicación: principios para su aplicación, integración y selección educativa. En J. Cabero \& J. Barroso (Coords.), Nuevos retos en tecnología educativa (pp. 41-67). Madrid: Síntesis.

Morales, E. (2014). Nuevos pobladores en el medio rural. Una apuesta por la sostenibilidad rural. El caso de Amayuelas da Abajo. En Asociación Española de Econo- 
Fernando Bumba, María Pilar Cáceres Reche, Miriam Ágreda Montoro

mía Agraria (Ed.), Territorios rurales, agriculturas locales y cadenas alimenticias. $X$ Coloquio Ibérico de Estudios Rurales. Ensino Primário (pp.319-322). España: Universitat Politècnica de València.

Organización de las Naciones Unidas para la Educación, la Ciencia y la Cultura. (2013). Objetivos del Desarrollo del Milenio. Informe de 2013. Nueva York: Naciones Unidas. Recuperado de http://www.un.org/es/millenniumgoals/pdf/ mdg-report-2013-spanish.pdf

Pedraza, M. A. (2011). La investigación-acción como vía de desarrollo profesional en el profesorado de educación física en la escuela rural (Tesis doctoral inédita). Universidad de Valladolid, España.

Raso, F. (2012). La escuela rural andaluza y su profesorado ante las Tecnologías de la Información y la Comunicación: estudio evaluativo (Tesis Doctoral). Granada: Universidad de Granada. Recuperado de http://hera.ugr.es/tesisugr/20771666.pdf

Raso, F., Aznar, I., \& Cáceres, M. P. (2014). Integración de Tecnologías de la Información y Comunicación: estudio evaluativo en la Escuela Rural Andaluza (España). Pixel-Bit: Revista de medios y educación, 45, 51-64.

Raso, F., Hinojo, M., \& Sola, J. M. (2015). Integración y uso docente de las tecnologías de la información y la comunicación (TIC) en la escuela rural de la provincia de Granada: estudio descriptivo. REICE. Revista Electrónica Iberoamericana sobre Calidad, Eficacia y Cambio en Educación, 13(1), 139-159.

Resenha social. (o9 de marzo de 2013). Abertura do ano lectivo no ensino superior marca semana. Agência Angola Press. Recuperado de http://www.angop.ao/ angola/pt_pt/noticias/sociedade/2013/2/10/Abertura-ano-lectivo-ensino-

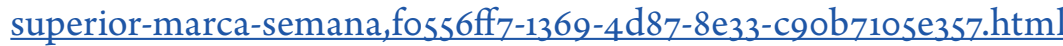

Sánchez, L. E., \& Navarro, M. G. J. (septiembre-diciembre, 2016). Secundarias rurales mediadas por tecnologías de la información y la comunicación en el norte de Argentina: democratización, inclusión y problemas éticos. Innovación Educativa, $15(69), 37-55$.

Trevizan, S. M. (septiembre, 2003). O que é rural? O que é urbano? Trabajo presentado en el Fórum Estadual de Educação do Campo (FEEC) e da Rede de Educação do Semi-Árido Brasileiro, preparatório para a Conferência Estadual do FEEC e RESAB, Bahia, Brasil. Recuperado de: http://www.forumeja.org.br/ec/files/ Texto\%2oSalvador\%2oTrevisan.pdf

Vázquez, R. (2016). Las escuelas públicas rurales: entre el bien común y la exclusión. Revista Interuniversitaria de Formación del Profesorado, 85(30.1), 67-79. Re- 


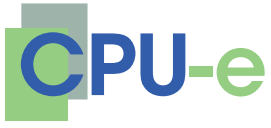

Estudio evaluativo de la escuela rural en

la provincia de Cabinda, Angola

Fernando Bumba, María Pilar Cáceres Reche, Miriam Ágreda Montoro

cuperado de https://www.researchgate.net/profile/Rosa_Vazquez-Recio/ publication/303459929_Las escuelas_publicas_rurales entre el bien comun_y_la_exclusion/links/5744319308aea45ee84d217a.pdf\#page $=68$ Zassala, C. (2003). Orientação Escolar e Profissional em Angola. Angola: Kulonga. Zassala, C. (2012). Orientación escolar y profesional en Angola. Luanda: Mayamba. 